\title{
Abdominal Obesity, Insulin Resistance, and Very Low-Density Lipoprotein Subclass Profile in Japanese School Children
}

\author{
Yuriko Abe1, Tomoo Okada1, 2*, Hiromi Okuma1, Minako Kazama1, Ryuta Yonezawa1, \\ Emiko Saito ${ }^{1}$, Yuki Kuromori ${ }^{1}$, Fujihiko Iwata ${ }^{1}$, Mitsuhiko Hara $^{1}$, Tatsuo Fuchigami ${ }^{1}$ and $^{1}$ \\ Shori Takahashi ${ }^{1}$ \\ ${ }^{1}$ Department of Paediatrics and Child Health, Nihon University School of Medicine, Tokyo, Japan \\ ${ }^{2}$ Department Nutrition and Life Science, Kanagawa Institute of Technology, Kanagawa, Japan
}

${ }^{*}$ Corresponding author: Tomoo Okada, Department of Pediatrics and Child Health, Nihon University School of Medicine30-1 Oyaguchi Kamicho, Itabashi-ku, Tokyo 173-8610, Japan, Tel: +81-3-3972-8111; Fax: +81-3-3957-6186; E-mail: tomo@med.email.ne.jp

Rec date: Jan 18, 2016 Acc date: Feb 26, 2016 Pub date: March 2, 2016

Copyright: (c) 2016 Abe Y, et al. This is an open-access article distributed under the terms of the Creative Commons Attribution License, which permits unrestricted use, distribution, and reproduction in any medium, provided the original author and source are credited.

\section{Abstract}

Background: Alterations in the very low-density lipoprotein (VLDL) subclass profile have been demonstrated in obese and insulin resistant adults; however, little information on VLDL subclasses is available in children. We determined the association between abdominal adiposity, insulin resistance, and triglyceride (TG) levels in each VLDL subclass in Japanese school children.

Methods: One hundred sixty-four children (79 boys and 85 girls), $10.9 \pm 1.6$ years of age (mean \pm SD), were recruited. We obtained the waist-to-height ratio (WHtR), lipid profile, and HOMA-R data from all children. The VLDL subclass profile was analyzed by HPLC.

Results: Children of either gender with abdominal obesity (WHtR $\geq 0.5$ ) had higher concentrations of large, medium, and small VLDL-TG in conjunction with the shift to larger size and elevated HOMA-R. The WHtR was positively related to concentrations of large (boys: $r=0.5800, p<$ 0.0001 ; girls: $r=0.3841, p=0.0003)$ and medium $(r=$ $0.5265, p<0.0001 ; r=0.3320, p=0.0019)$ VLDL-TG. The small VLDL-TG concentration was positively correlated to the WHtR in boys $(r=0.4902, p<0.0001)$, but not girls. In multiple regression analyses, the WHtR was an independent determinant of large, medium, and small VLDL-TG concentrations in boys, but not in girls, taking HOMA-R into account.

Conclusions: Abdominal obesity was associated with the VLDL subclass profile in Japanese school children; however, the impact of abdominal adiposity on the VLDL subclass alteration demonstrated a gender difference.

Keywords: Obesity; Insulin resistance; Japanese school children

\section{Introduction}

Childhood obesity is one of the most serious public health problems worldwide. Obese children, especially children who have abdominal obesity, are likely to develop metabolic syndrome (MetS) during childhood [1-3], which is defined by the clustering of several metabolic abnormalities, such as dyslipidemia, hypertension, and hyperglycemia, and is associated with a higher risk of cardiovascular diseases and type 2 diabetes in later life [4,5]. In Japan, cross-sectional analysis showed that the prevalence of childhood obesity has gradually decreased since the early 2000s, with the highest prevalence in the late 1990 s to early 2000s; [6] however, the prevalence of MetS is not lower in pre-teen Japanese children who are overweight in comparison with overweight adolescents in the United States [7]. In a cohort study of Japanese adults without MetS, new onset of MetS was observed in $9.32 \%$ over a 5 -year period, and the presence of obesity and a high triglycerides (TG) level at baseline was associated with the highest hazard ratio for risk to develop MetS [8]. Furthermore, in Japanese men, an increased number of very low-density lipoprotein (VLDL) particles is strongly associated with coronary heart disease, independent of intermediate-density lipoprotein or small, dense low-density lipoprotein (LDL) [9].

VLDL particles are heterogeneous in size, density, and composition. In adult studies, central obesity has been reported to be strongly associated with larger VLDL subclasses $[10,11]$; however, little information on VLDL subclasses in children is available. Based on the Bogalusa Heart Study, [12] the relationship between obesity and specific VLDL subclasses was investigated in black and white children, and waist circumference was shown to be associated with large VLDL, but not with small VLDL. In addition, racial differences were observed; specifically, the concentration of large VLDL in white children was 2.5 times higher than black children and showed a 6-fold stronger association with waist circumference in white children. Furthermore, insulin resistance is an independent factor determining the VLDL subclass profile $[13,14]$. Insulin- 
resistant children have higher VLDL concentrations, and visceral adiposity, race, and insulin resistance are independent determinants of VLDL size [15]. Taken together, these studies have shown that the relationships between abdominal adiposity, insulin resistance, and VLDL subclass profile in children depend on the characteristics of the study population, especially ethnicity. In the present study, we analyzed VLDL subclasses in Japanese school children and investigated the association between abdominal adiposity, insulin resistance, and TG levels in each VLDL subclass.

\section{Methods}

The subjects were 164 children (79 boys and 85 girls), $10.9 \pm$ 1.6 years of age (mean \pm SD; range $=9-13$ years) who attended one of the two schools selected for this study and participated voluntarily in this study. All of the children were free from diseases other than dyslipidemia or obesity. Each child's standing height and weight were measured, and the body mass index (BMI; $\mathrm{kg} / \mathrm{m}^{2}$ ) was calculated. Obesity is defined as having a percentage of overweight greater than $20 \%$, calculated according to the standard weight for sex, age and height: ((body weight - standard weight)/standard weight) $X$ 100 [16]. Waist circumference was measured at the level of the umbilicus, and the waist-to-height ratio (WHtR) was calculated. Abdominal obesity is defined as a WHtR $\geq 0.5$ [17].

Blood samples were obtained in the morning after an overnight fast. Total cholesterol (TC) and TG concentrations were measured by enzymatic methods. Serum lipoprotein analyses were performed by HPLC with gel permeation columns (LipoSEARCH; Skylight-Biotec, Inc., Akita, Japan), and LDL-cholesterol (C) and high-density lipoprotein-cholesterol (HDL-C) concentrations, and TG concentrations in three VLDL subclasses (large, medium, and small) were measured [18]. Non-HDL-C is defined as TC minus HDL-C. Plasma insulin and glucose concentrations were determined, and the homeostasis model of assessment ratio (HOMA-R) was obtained using Matthews' formula as an index of insulin resistance [19].

In the present study, we employed the diagnostic criteria of MetS in Japanese children, as having abdominal obesity (waist circumference $\geq 80 \mathrm{~cm}$ and/or $\mathrm{WHtR} \geq 0.5$ ) plus two or more of the following: (i) dyslipidemia: high TG $\geq 120 \mathrm{mg} / \mathrm{dl}$ and/or low HDL-C $<40 \mathrm{mg} / \mathrm{dl}$; (ii) elevated SBP ( $\geq 125 \mathrm{mmHg}$ ) and/or DBP ( $\geq 70 \mathrm{mmHg}$ ); (iii) elevated fasting glucose level ( $\geq 100$ $\mathrm{mg} / \mathrm{dl}$ ). In case of 6 grade or younger school children, the cutoff value of waist circumference is $75 \mathrm{~cm} \mathrm{[20].}$
Informed consent was obtained from each child and the child's parents. The study protocol was approved by the local Ethics Committee, which consists of members of the school's Health Education Committee. The Health Education Committee also includes members of the local Board of Education and representatives from Nihon University Itabashi Hospital.

All of the data are expressed as the mean \pm SD. The group differences were assessed using an unpaired t-test. Single and multiple linear regression analyses were used to assess the correlation between variables. A P-value $<0.05$ was considered statistically significant. All of the statistical analyses were conducted using the JMP statistical package (v9.0; SAS Institute, Inc., Cary, NC, USA).

\section{Results}

Ten boys (12.7\%) and eleven girls (12.9\%) were diagnosed as obesity, and thirteen boys (16.5\%) and nine girls (10.6\%) had abdominal obesity. Compared to children without abdominal obesity, children with abdominal obesity exhibited higher TG and insulin concentrations and HOMA-R, and lower HDL-C concentrations, regardless of gender. LDL-C and non-HDL-C concentrations in boys with abdominal obesity were also higher than boys without abdominal obesity; however, no difference was observed in LDL-C or non-HDL-C concentrations in girls with and without abdominal obesity.

In children without abdominal obesity, girls had higher TC, LDL-C, TG, non-HDL-C, insulin concentrations, and HOMA-R than boys. In the study subjects, we found ten boys and twenty-eight girls with insulin resistance (HOMA-R $\geq 2.5$ ), and only one boy with metabolic syndrome.

\section{VLDL subclass profile measured using the HPLC method}

Children with abdominal obesity had higher large, medium, and small VLDL-TG concentrations than children without abdominal obesity, regardless of gender (Table 1).

In children without abdominal obesity, girls had higher medium VLDL-TG and small VLDL-TG concentrations than boys. In children with abdominal obesity, the TG distribution in VLDL was shifted to a larger subclass in boys and girls.

Table 1: Characteristics of the subjects.

\begin{tabular}{|c|c|c|c|c|c|c|c|c|c|c|}
\hline \multicolumn{6}{|l|}{ Boys } & \multicolumn{5}{|l|}{ Girls } \\
\hline \multicolumn{6}{|l|}{$n=79$} & \multicolumn{5}{|l|}{$n=85$} \\
\hline & $\begin{array}{l}\text { without } \\
\text { obesity }\end{array}$ & abdominal & $\begin{array}{l}\text { with } \\
\text { obesity }\end{array}$ & abdominal & $p$ value & $\begin{array}{l}\text { without } \\
\text { obesity }\end{array}$ & abdominal & $\begin{array}{l}\text { with } \\
\text { obesity }\end{array}$ & abdominal & $p$ value \\
\hline & $n=66$ & & $n=13$ & & & $n=76$ & & $\mathrm{n}=9$ & & \\
\hline Age (year) & $11.0 \pm 1.6$ & & $10.8 \pm 1.9$ & & 0.6176 & $10.8 \pm 1.6$ & & $11.7 \pm 1.0$ & & 0.1251 \\
\hline
\end{tabular}




\begin{tabular}{|c|c|c|c|c|c|c|c|c|c|c|}
\hline Height (cm) & $146.1 \pm 12.9$ & & $\begin{array}{ll}146.5 & \pm \\
12.5 & \end{array}$ & & 0.9231 & $142.3 \pm 10.8$ & & $150.5 \pm 6.9$ & & 0.0285 \\
\hline Body weight $(\mathrm{kg})$ & $37.3 \pm 9.7$ & & $\begin{array}{l}52.1 \quad \pm \\
14.8\end{array}$ & & $<0.0001$ & $35.6 \pm 9.3$ & & $59.6 \pm 9.5$ & & $<0.0001$ \\
\hline $\begin{array}{l}\text { Waist circumference } \\
(\mathrm{cm})\end{array}$ & $60.0 \pm 6.0$ & & $82.3 \pm 9.4$ & & $<0.0001$ & $59.8 \pm 6.5$ & & $80.1 \pm 3.5$ & & $<0.0001$ \\
\hline Body mass index & $17.1 \pm 1.9$ & & $23.9 \pm 3.6$ & & $<0.0001$ & $17.3 \pm 2.5$ & & $26.1 \pm 2.2$ & & $<0.0001$ \\
\hline Waist/height ratio & $0.41 \pm 0.03$ & & $\begin{array}{l}0.56 \quad \pm \\
0.04\end{array}$ & & $<0.0001$ & $0.42 \pm 0.03$ & & $0.53 \pm 0.02$ & & $<0.0001$ \\
\hline $\begin{array}{l}\text { Percent } \\
\text { overweight (\%) }\end{array}$ & $-5.3 \pm 8.7$ & & $\begin{array}{ll}31.7 & \pm \\
18.1 & \end{array}$ & & $<0.0001$ & $-3.3 \pm 12.0$ & & $38.7 \pm 6.3$ & & $<0.0001$ \\
\hline $\begin{array}{l}\text { Total } \\
(\mathrm{mg} / \mathrm{dl})\end{array} \quad$ cholesterol & $168.5 \pm 21.1$ & & $\begin{array}{ll}173.8 & \pm \\
28.3 & \end{array}$ & & 0.4422 & $176.9 \pm 22.0^{*}$ & & $\begin{array}{ll}162.6 & \pm \\
24.8 & \end{array}$ & & 0.0716 \\
\hline $\operatorname{LDLC}(\mathrm{mg} / \mathrm{dl})$ & $90.9 \pm 17.2$ & & $\begin{array}{l}105.2 \quad \pm \\
25.6\end{array}$ & & 0.0142 & $99.7 \pm 20.1^{* *}$ & & $96.0 \pm 22.1$ & & 0.61 \\
\hline $\mathrm{HDLC}(\mathrm{mg} / \mathrm{dl})$ & $69.2 \pm 12.6$ & & $53.5 \pm 9.6$ & & $<0.0001$ & $66.9 \pm 11.3$ & & $49.8 \pm 8.5$ & & $<0.0001$ \\
\hline Triglyceride (mg/dl) & $42.0 \pm 17.4$ & & $\begin{array}{l}75.7 \quad \pm \\
27.1\end{array}$ & & $<0.0001$ & $51.5 \pm 2.9^{* *}$ & & $83.8 \pm 8.3$ & & 0.0004 \\
\hline $\begin{array}{l}\text { Non-HDL cholesterol } \\
(\mathrm{mg} / \mathrm{dl})\end{array}$ & $99.3 \pm 18.1$ & & $\begin{array}{l}120.3 \quad \pm \\
26.1\end{array}$ & & 0.0007 & $\begin{array}{ll}110.0 & \pm \\
20.7^{* *} & \end{array}$ & & $\begin{array}{ll}112.8 & \pm \\
27.1 & \end{array}$ & & 0.7114 \\
\hline 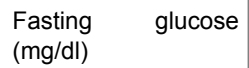 & $88.7 \pm 4.9$ & & $91.7 \pm 5.0$ & & 0.0499 & $87.5 \pm 6.2$ & & $89.9 \pm 5.1$ & & 0.2664 \\
\hline Insulin $(\mu \mathrm{U} / \mathrm{ml})$ & $6.1 \pm 2.9$ & & $12.1 \pm 6.4$ & & $<0.0001$ & $8.4 \pm 4.8^{* * *}$ & & $18.5 \pm 7.5$ & & $<0.0001$ \\
\hline HOMA-R & $1.3 \pm 0.7$ & & $2.8 \pm 1.6$ & & $<0.0001$ & $1.8 \pm 1.1^{* *}$ & & $4.1 \pm 1.9$ & & $<0.0001$ \\
\hline $\begin{array}{l}\text { VLDL-TG } \quad(\mathrm{mg} / \mathrm{dl}) \\
(\%)\end{array}$ & $20.1 \pm 14.0$ & $100.0 \%$ & $\begin{array}{ll}45.9 & \pm \\
23.2 & \end{array}$ & $100.0 \%$ & $<0.0001$ & $26.0 \pm 19.1^{*}$ & $100.0 \%$ & $54.1 \pm 27.3$ & $100.0 \%$ & 0.0001 \\
\hline $\begin{array}{l}\text { Large VLDL-TG } \\
(\mathrm{mg} / \mathrm{dl})(\%)\end{array}$ & $8.6 \pm 7.3$ & $42.8 \%$ & $\begin{array}{ll}23.7 & \pm \\
14.3 & \end{array}$ & $51.6 \%$ & $<0.0001$ & $11.2 \pm 10.6$ & $43.1 \%$ & $27.5 \pm 16.3$ & $50.8 \%$ & $<0.0001$ \\
\hline $\begin{array}{l}\text { Medium VLDL-TG } \\
(\mathrm{mg} / \mathrm{dl})(\%)\end{array}$ & $7.5 \pm 5.1$ & $37.3 \%$ & $15.5 \pm 7.6$ & $33.8 \%$ & $<0.0001$ & $9.8 \pm 6.9^{*}$ & $37.7 \%$ & $18.9 \pm 8.9$ & $34.9 \%$ & 0.0005 \\
\hline $\begin{array}{l}\text { Small VLDL-TG } \\
(\mathrm{mg} / \mathrm{dl})(\%)\end{array}$ & $4.0 \pm 1.9$ & $4.0 \pm 1.9$ & $6.8 \pm 2.3$ & $14.6 \%$ & $<0.0001$ & $4.9 \pm 2.3^{* *}$ & $19.2 \%$ & $7.7 \pm 2.8$ & $14.3 \%$ & 0.0014 \\
\hline \multicolumn{11}{|l|}{ Mean $\pm S D$} \\
\hline \multicolumn{11}{|c|}{ Boys vs. Girls; ${ }^{*}: p<0.05,{ }^{* *} p<0.01,{ }^{* * *}: p<0.001$} \\
\hline
\end{tabular}

\section{Relationship between TG concentration in each VLDL subclass and variables}

Total VLDL-TG concentration had a significant positive relationship with WHtR (boys: $r=0.5662, p<0.0001$; girls: $r=$ 0.3693, $p=0.0005)$ and HOMA-R $(r=0.5184, p<0.0001 ; r=$ $0.6141, p<0.0001)$.

The relationship between TG concentration in each VLDL subclass and WHtR and HOMA-R were analyzed using single regression analyses (Table 2 ).
WHtR was positively related to large (boys: $r=0.5800, p<$ 0.0001; girls: $r=0.3841, p=0.0003)$ and medium $(r=0.5265, p$ $<0.0001 ; r=0.3320, p=0.0019)$ VLDL-TG regardless of gender.

The small VLDL-TG concentration was positively correlated with the WHtR in boys $(r=0.4902, p<0.0001)$, but not girls. HOMA-R had significantly positive relationships with large (boys: $r=0.4822, p<0.0001$; girls: $r=0.6141, p<0.0001$ ) and medium ( $r=0.5282, p<0.0001 ; r=0.5831, p<0.0001$ ) VLDLTG in both genders.

The small VLDL-TG concentration was positively correlated with HOMA-R in boys $(r=0.5458, p<0.0001)$, but not girls. 
Based on multiple regression analyses (Table 3), both the WHtR and HOMA-R were independent determinants of large, medium, and small VLDL-TG concentrations in boys.

Table 2: Relationship between WHtR, HOMA-R and VLDL-TG.
In contrast, HOMA-R was an independent determinant of all VLDL-TG subclasses in girls, unlike the WHtR.

\begin{tabular}{|c|c|c|c|c|}
\hline Variables & & & & \\
\hline Boys & $r$ & $\mathrm{p}$ & $r$ & $\mathrm{p}$ \\
\hline VLDL-TG & 0.5662 & $<0.0001$ & 0.5184 & $<0.0001$ \\
\hline large VLDL-TG & 0.5800 & $<0.0001$ & 0.4822 & $<0.0001$ \\
\hline medium VLDL-TG & 0.5265 & $<0.0001$ & 0.5282 & $<0.0001$ \\
\hline small VLDL-TG & 0.4902 & $<0.0001$ & 0.5458 & $<0.0001$ \\
\hline Girls & $\mathrm{r}$ & $\mathrm{p}$ & $r$ & $p$ \\
\hline VLDL-TG & 0.3693 & 0.0005 & 0.6141 & $<0.0001$ \\
\hline large VLDL-TG & 0.3841 & 0.0003 & 0.6141 & $<0.0001$ \\
\hline medium VLDL-TG & 0.3320 & 0.0019 & 0.5831 & $<0.0001$ \\
\hline small VLDL-TG & 0.0263 & 0.8112 & 0.2047 & 0.0603 \\
\hline
\end{tabular}

Table 3: Multiple regression analysis of the relationship between VLDL subclass profile and WHtR and HOMA-R.

\begin{tabular}{|c|c|c|c|c|c|c|c|c|c|}
\hline \multirow{2}{*}{$\begin{array}{l}\text { Dependent } \\
\text { variables }\end{array}$} & \multirow{2}{*}{$\begin{array}{l}\text { Independent } \\
\text { variables }\end{array}$} & \multicolumn{4}{|l|}{ Boys } & \multicolumn{4}{|c|}{ Girls } \\
\hline & & $\mathrm{r} 2$ & $\beta$ & s.e. & $\mathbf{p}$ & r2 & $\beta$ & s.e. & $\mathbf{p}$ \\
\hline \multirow[t]{2}{*}{ VLDL-TG } & WHtR & 0.38 & 117 & 31.31 & 0 & 0.4 & -6.54 & 51.51 & 0.9 \\
\hline & \multicolumn{2}{|l|}{ HOMA-R } & 5.3 & 1.938 & 0 & & 9.817 & 17.43 & $<0.0001$ \\
\hline \multirow[t]{2}{*}{ large VLDL-TG } & WHtR & 0.37 & 74 & 17.76 & $<0.0001$ & 0.4 & 2.575 & 29.09 & 0.93 \\
\hline & \multicolumn{2}{|l|}{ HOMA-R } & 2.4 & 1.1 & 0 & & 5.412 & 0.985 & $<0.0001$ \\
\hline \multirow{2}{*}{$\begin{array}{l}\text { medium VLDL- } \\
\text { TG }\end{array}$} & WHtR & 0.36 & 33 & 10.91 & 0 & 0.3 & -7.08 & 18.53 & 0.7 \\
\hline & \multicolumn{2}{|l|}{ HOMA-R } & 2.1 & 0.675 & 0 & & 3.362 & 0.627 & $<0.0001$ \\
\hline \multirow[t]{2}{*}{ small VLDL-TG } & WHtR & 0.35 & 9.5 & 3.867 & 0 & 0.3 & -2.04 & 6.247 & 0.75 \\
\hline & \multicolumn{2}{|l|}{ HOMA-R } & 0.9 & 0.239 & 0 & & 1.042 & 0.211 & $<0.0001$ \\
\hline
\end{tabular}

\section{Discussion}

Japanese school children with abdominal obesity, both boys and girls, had elevated concentrations of large, medium, and small VLDL-TG, as well as insulin resistance. In addition, abdominal adiposity was a significant independent determinant of the VLDL subclass profile in boys, but not girls. HOMA-R was a significant independent determinant of VLDL subclass profile in boys and girls.

Previously, the relationship between waist circumferences and VLDL subclasses has been investigated in children, demonstrating that waist circumference is associated with large VLDL, but not small VLDL [12]. In another study, obese adolescents with intrahepatic fat accumulation had a marked increase in large VLDL, and to a lesser extent, medium VLDL, but no differences in small VLDL compared with obese adolescents without hepatic fat accumulation [21]. In the present study, we confirmed that Japanese school children with abdominal obesity, both boys and girls, had elevated concentrations in VLDL-TG, and the subclass distribution was shifted to larger particles.

It is known that hepatic fatty acid availability and insulin are two major modulators of VLDL production and secretion [22]. The acute effects of insulin on VLDL kinetics have been investigated in healthy human subjects [23]. Insulin infusion down-regulates VLDL1 secretion and increases VLDL2 secretion, resulting in a shift in the balance from VLDL1 to VLDL2; however, in an insulin resistance state, hyperinsulinemia promotes lipogenesis and suppression of VLDL1 secretion is impaired. Our results regarding alterations 
in the VLDL subclass profile observed in children with abdominal obesity and the relationship between HOMA-R and VLDL subclass are consistent with the underlying mechanism. Therefore, insulin resistance is an important factor determining VLDL subclass profile in Japanese school children.

Another important finding of the present study was the gender difference in the relationship between abdominal adiposity and VLDL-TG subclass distribution. The previous study investigating the impact of abdominal adiposity on VLDL subclass profile in black and white children demonstrated the racial difference; however, the gender difference was not evaluated [12]. Because of the hyperlipolytic state, the liver of abdominal obese patients is exposed to an increased flux of adipose-derived fatty acids, which contributes to an increased production and secretion of VLDL particles. In a human adult study, gender and obesity were shown to have independent and different effects on VLDL-TG kinetics [24]. In lean healthy subjects, women have a higher rate of VLDL-TG production and a higher rate of VLDL-TG clearance than men. In obese subjects, the rate of VLDL-TG production increases in men, while no change occurs in women, and the rate of VLDL-TG clearance decreases in women, while no change occurs in men. Thus, the plasma VLDL-TG concentration in obese adults is determined primarily by the production rate in men, but by the clearance rate in women. The reduced VLDL-TG clearance is associated with insulin resistance. A human adult study has demonstrated that muscle LPL activity is correlated with the serum TG concentration, and is also related to the fasting insulin concentration and insulin sensitivity, suggesting that the hyperinsulinemia associated with insulin resistance may have a down-regulating effect on muscle LPL activity [25]. In addition, estrogen may decrease lipoprotein lipase activity by a post-transcriptional modification of protein levels in premenopausal women [26]. Our results in Japanese girls demonstrate that HOMA-R, but not the WHtR, is an independent determinant of all VLDL-TG subclasses based on multiple regression analyses, suggesting that impaired insulin actions, rather than an increased flux of fatty acids, is a main mechanism explaining TG elevation combined with the changes in VLDL-TG subclass profile in girls. In our previous study investing HDL-C subclass alteration in abdominal obese children [27], gender difference was also demonstrated; cholesterol levels in small and very small HDL were lower in girls than boys. Furthermore, we evaluated the LDL subclass profile in this study subjects, showing that LDL subclass alteration associated with abdominal obesity was exhibited in boys, but not in girls (Supplementary table). The sex hormonal status should be evaluated in future studies.

Our study had limitations. We investigated VLDL subclasses using HPLC methods. Therefore, we could not compare our data with previous reports that used other methods, $[12,15,21]$ and the racial difference regarding the impact of abdominal obesity on the alteration in VLDL subclass profile could not be analyzed; however, we confirmed that the VLDL subclass profile was altered in Japanese school children with abdominal obesity. Second, we could not obtain information on nutritional intake. Dietary intake, especially fish consumption, is related to a favorable subclass distribution of
VLDL $[28,29]$. Further detailed studies, including dietary assessment, should be conducted to elucidate the mechanisms of VLDL subclass alterations associated with abdominal obesity.

\section{Conclusions}

It is now recognized that a fundamental defect in the dyslipidemia component of MetS is an overproduction of large VLDL particles, resulting in a higher concentration of small dense LDL and a lower concentration of HDL-C.20 In Japanese school children, we found that abdominal obesity and insulin resistance were associated with the VLDL subclass profile, which suggests that VLDL subclass analysis may provide a better guide for the management of MetS, even in school children.

\section{Acknowledgments}

Preparation of this article was partly supported by a grant from Health and Labour Sciences Research Grants: Comprehensive Research on Cardiovascular Diseases, no 17160501 in Japan, which was entitled 'Cohort study for concept, pathophysiology, establishment of diagnostic criteria, and effective intervention for metabolic syndrome in childhood'.

\section{Author Disclosure Statement}

No competing financial interests exist.

\section{References}

1. Weiss R, Dziura J, Burgert TS (2004) Obesity and the metabolic syndrome in children and adolescents. N Engl J Med 350: 2362-2374.

2. Flynn MA, McNeil DA, Maloff B (2006) Reducing obesity and related chronic disease risk in children and youth: a synthesis of evidence with 'best practice' recommendations. Obes Rev 7: 7-66.

3. Han JC, Lawlor DA, Kimm SY (2010) Childhood obesity. Lancet 375: $1737-1748$

4. Després JP, Lemieux I, Bergeron J (2008) Abdominal obesity and the metabolic syndrome: contribution to global cardiometabolic risk. Arterioscler Thromb Vasc Biol 28: 1039-1049.

5. Bergman RN, Kim SP, Hsu IR (2007) Abdominal obesity: role in the pathophysiology of metabolic disease and cardiovascular risk. Am J Med.120: S3-8.

6. Yoshinaga M, Ichiki T, Tanaka Y (2010) Prevalence of childhood obesity from 1978 to 2007 in Japan. Pediatr Int 52: 213-217.

7. Yoshinaga M, Tanaka S, Shimago A (2005) Metabolic syndrome in overweight and obese Japanese children. Obes Res 13: $1135-1140$

8. Suzuki A, Kosuge K, Nanyu O (2010) Five year study of cardiovascular risk factors in Japanese people: implications concerning new onset of metabolic syndrome. Intern Med 49: $1-6$ 
9. Koba S, Hirano T, Sakaue T (2002) An increased number of very low-density lipoprotein particles is strongly associated with coronary heart disease in Japanese men, independently of intermediate-density lipoprotein or low-density lipoprotein. Coron Artery Dis 13: 255-262.

10. James RW, Brulhart-Meyner MC, Lehman T (1997) Lipoprotein distribution and composition in obesity: their association with central adiposity. Int J Obes Relat Metab Disord 21: 1115-1120.

11. Terry RB, Wood PD, Haskell WL (1989) Regional adiposity patterns in relation to lipids, lipoprotein cholesterol, and lipoprotein subfraction mass in men. J Clin Endocrinol Metab 68: 191-199.

12. Freedman DS, Bowman BA, Otvos JD (2002) Differences in the relation of obesity to serum triacylglycerol and VLDL subclass concentrations between black and white children: the Bogalusa Heart Study. Am J Clin Nutr 75: 827-833.

13. Wang J, Stančáková A, Soininen P (2012) Lipoprotein subclass profiles in individuals with varying degrees of glucose tolerance: a population-based study of 9399 Finnish men. J Intern Med 272: $562-672$

14. Goff DC Jr, D'Agostino RB Jr, Haffner SM (2005) Insulin resistance and adiposity influence lipoprotein size and subclass concentrations. Results from the Insulin Resistance Atherosclerosis Study. Metabolism 54: 264-270.

15. Burns SF, Lee S, Arslanian SA (2009) In vivo insulin sensitivity and lipoprotein particle size and concentration in black and white children. Diabetes Care 32: 2087-2093.

16. Ikiuo K, Hashimoto R, Murata M (2010) Discussion on the new physical fitness definition in School Health Program - On the comparison between a new and a previous definition for the physical fitness of school aged children and the secular trend of the prevalence of obesity and thinness in them from 1980 to 2000. J Child Health 69: 6-13.

17. Hara M, Saitou E, Iwata F (2002) Waist-to-height ratio is the best predictor of cardiovascular disease risk factors in Japanese school children. J Atheroscler Thromb 9: 127-132.

18. Usui S, Hara Y, Hosaki S (2002) A new on-line dual enzymatic method for simultaneous quantification of cholesterol and triglycerides in lipoproteins by HPLC. J Lipid Res 43: 805-814.
19. Matthews DR, Hosker JP, Rudenski AS (1985) Homeostasis model assessment: insulin resistance and beta-cell function from fasting plasma glucose and insulin concentrations in man. Diabetologia 28: 412-419.

20. Abe Y, Kikuchi T, Nagasaki K (2009) Usefulness of GPT for diagnosis of metabolic syndrome in obese Japanese children. J Atheroscler Thromb 16: 902-909.

21. Cali AM, Zern TL, Taksali SE (2007) Intrahepatic fat accumulation and alterations in lipoprotein composition in obese adolescents: a perfect proatherogenic state. Diabetes Care 30: 3093-3098.

22. Adiels M, Olofsson SO, Taskinen MR (2008) Overproduction of very low-density lipoproteins is the hallmark of the dyslipidemia in the metabolic syndrome. Arterioscler Thromb Vasc Biol 28: $1225-1236$.

23. Adiels M, Westerbacka J, Soro-Paavonen A (2007) Acute suppression of VLDL1 secretion rate by insulin is associated with hepatic fat content and insulin resistance. Diabetologia 50: 2356-2365.

24. Mittendorfer B, Patterson BW, Klein S (2003) Effect of sex and obesity on basal VLDL-triacylglycerol kinetics. Am J Clin Nutr 77: 573-279.

25. Pollare T, Vessby B, Lithell H (1991) Lipoprotein lipase activity in skeletal muscle is related to insulin sensitivity. Arterioscler Thromb.11: 1192-1203.

26. Price TM, O'Brien SN, Welter BH (1998) Estrogen regulation of adipose tissue lipoprotein lipase-possible mechanism of body fat distribution. Am J Obstet Gynecol 178: 101-107.

27. Okuma H, Okada T, Abe Y (2013) Abdominal adiposity is associated with high-density lipoprotein subclasses in Japanese school children. Clin Chim Acta 425: 80-84.

28. Annuzzi G, Rivellese AA, Wang H (2012) Lipoprotein subfractions and dietary intake of n-3 fatty acid: the Genetics of Coronary Artery Disease in Alaska Natives study. Am J Clin Nutr 95: 1315-1322.

29. Bogl LH, Pietiläinen KH, Rissanen A (2013) Association between habitual dietary intake and lipoprotein subclass profile in healthy young adults. Nutr Metab Cardiovasc Dis 23: 1071-1078. 